\title{
HIV-1 resistance testing influences treatment decision-making
}

\begin{abstract}
Objective: To investigates how the use of HIV-1 resistance tests influences physician decision-making. Methods: Ten experienced reference physicians from the Brazilian Network for Drug Resistance each received ten patients' case histories. The selected patients had experienced at least two virological failures. First, reference physicians were asked to empirically select a new regimen for each patient. Second, after genotype report (ViroSeq 2.6) was provided, and physicians were again asked to select a new regimen considering this additional information. Finally, they were asked to select a regimen after receiving a virtual phenotype result (vircoTYPE 3.9.00). Results: In 79\% of the cases, physicians changed their empirical choice of regimen after receiving the genotype report, resulting in an increase in the mean number of active drugs from 1.8 to $2.2(\mathrm{p}=0.0003)$, while the average number of drugs/regimen remained at 4.0. After receipt of the virtual phenotype report, additional changes were made in $75 \%$ of the patient cases, resulting in an increase in the number of active drugs to $2.8(\mathrm{p}<0.0001)$, while the average number of drugs/ regimen remained at 4.0. After receipt of the genotype report, $48 \%$ of the changes were in NRTIs, $29 \%$ were in NNRTIs and 60\% were in PIs; after consideration of the virtual phenotype, $61 \%, 10 \%$ and $49 \%$ of the changes, respectively, were in these categories of drugs. Fourteen percent of the physicians rated the genotype report as "extremely useful", whereas 34\% rated the subsequent virtual phenotype report as "extremely useful" ( $p=0.0003)$. Conclusions: Resistance testing has a significant impact on physicians' choices of antiretroviral salvage therapies, and it promotes the selection of more active drugs.
\end{abstract}

Keywords: genotype, virtual phenotype, antiretroviral resistance, Brazil.

[Braz J Infect Dis 2010;14(5):489-494] @Elsevier Editora Ltda.

\section{INTRODUCTION}

Although antiretroviral therapy provides significant reductions in HIV-related mortality and morbidity, these reductions may be limited in duration. ${ }^{1}$ In some developing countries where antiretrovirals are widely available, one study showed that the median time to loss of treatment benefit was approximately 14 months among treatment-naïve patients. ${ }^{2}$ As a result of antiretroviral failure, resistance has been increasingly detected, ${ }^{3}$ even in developing countries. An analysis of 791 samples submitted for resistance testing in Brazil revealed that $96.6 \%$ of the samples had primary resistance-associated mutations present in the reverse transcriptase coding region, and $90.3 \%$ had mutations in the protease coding region. Multi-drug resistance was common, with $36.8 \%$ of samples revealing resistance to at least one drug from each therapeutic class. ${ }^{4}$ Furthermore, antiretroviral resistance has been associated with disease progression and death. ${ }^{5,6}$

Prospective randomized clinical trials have shown that, among patients on salvage therapy, the selection of antiretrovirals with the aid of resistance testing results in improved virological response. ${ }^{7-11}$ Furthermore, studies have shown that the combination of expert advice and genotype testing further improves virologic outcomes among patients on salvage therapy. ${ }^{10}$ However, an understanding of how resistance testing influences decision-making by individual physicians has not been established.

The Brazilian Network for Antiretroviral Resistance is comprised of more than 100 trained expert physicians (designated 'reference physicians') who analyze the results of genotypic tests performed in the Brazilian public system and make recommendations to treating physicians. These reference physicians are trained to act as clinical virologists for HIV antiretroviral salvage therapy. In the Brazilian Network for Antiretroviral Resistsicians are analyzed by reference physicians, and salvage regimen recommendations made by the reference physicians are delivered to the attending physicians with the test results to optimize ance, genotype tests ordered by attending phy-
Author

Ricardo Sobhie Diaz ${ }^{1}$

Maria Cecilia A Sucupira ${ }^{1}$

Tania RC Vergara ${ }^{1}$

Carlos Brites $^{2}$

Rosana Del Bianco

Francisco Bonasser Filho ${ }^{3}$

Geova Keny B Colares ${ }^{4}$

Estevão Portela ${ }^{5}$

Lia Adler Cherman ${ }^{6}$

Nemora Tregnago Barcelos

Unai Tupinambas ${ }^{8}$

Gilberto Turcato Jr. ${ }^{1}$

Lisa Allamasey ${ }^{9}$

Lee Bacheler ${ }^{10}$

Martin Tuohy9

Brazilian Network

Reference Physicians

Working Group ${ }^{6}$

${ }^{1}$ Universidade Federal de São Paulo, São Paulo, Brazil.

${ }^{2}$ Universidade Federal de

Bahia, Brazil.

${ }^{3}$ Hospital Emilio Ribas, São

Paulo, SP, Brazil.

${ }^{4}$ Universidade de Fortaleza,

CE, Brazil.

${ }^{5}$ Fundação Oswaldo Cruz,

RJ, Brazil.

${ }^{6}$ Network Reference

Physicians Working Group.

${ }^{7}$ Secretaria Estadual de

Saúde do Rio Grande do

Sul, RS, Brazil.

${ }^{8}$ Universidade Federal de

Minas Gerais, MG, Brasil.

${ }^{9}$ Virco BVBA, Mechelen,

Belgium.

${ }^{10}$ VircoLab Inc, Durham,

NC, USA.

Submitted on: 03/05/2010 Approved on: 08/04/2010

Correspondence to: Ricardo Sobhie Diaz, M.D., PhD

Retrovirology Laboratory,

Universidade Federal de

São Paulo - EPM

R. Pedro de Toledo, 781, Sao

Paulo, SP - 04039 - Brazil

Phone: +55-110-9109-0445

Phone/fax: +55-11-5579-

8226, +55-11-5571-2130

+55-11-5084-4262

Email: rsdiaz@catg.com.br

Financial disclosure:

Dr Diaz also works at

Laboratorio Centro

de Genomas which

commercializes resistance

HIV tests including

genotype and virtual

phenotype. 
the efficacy of salvage regimens. In this study, we evaluated the impact of two different genotypic analysis systems, ViroSeq 2.6 and Virtual Phenotype (VircoTYPE HIV-1, 3.9.00), on the choice of antiretroviral salvage therapy suggested by reference physicians of the Brazilian Network for Antiretroviral Resistance.

\section{METHODS}

Ten genotyping reference physicians from the Brazilian Network for Antiretroviral Resistance were selected to participate in the study. The reference physicians were selected on the basis of level of experience, the most experienced physicians being chosen. The selected doctors had been acting as reference physicians for at least 5 years and treating HIV/AIDS for at least 10 years. Training in salvage therapy and interpretation of resistance tests for the reference physicians occurs annually in a four-day workshop organized exclusively for this purpose. Five out of the ten chosen physicians play a key role in the preparation of the training workshops for the network of reference physicians. The reference physicians were selected from different Brazilian states based on the prevalence of AIDS cases in each state: three doctors from São Paulo (SP), three from Rio de Janeiro (RJ), one from Ribeirão Preto (SP), one from Belo Horizonte $(\mathrm{MG})$, one from Salvador (BA) and one from Porto Alegre (RS). One hundred consecutive patients presenting with virologic failure were selected based on the availability of both a genotype (ViroSeq 2.6, Celera Diagnostics, Alameda, California, USA) and virtual phenotype (VircoTYPE HIV-1, 3.9.00), reports. All patients were on antiretrovirals at the time plasma samples were collected for genotyping. Patients evaluated in this study were selected from the metropolitan area of São Paulo (SP), and all the laboratory work was performed at the Retrovirology Laboratory of the Universidade Federal de São Paulo, Brazil.

The study goals were (a) to observe the decision-making process and (b) to evaluate the antiretroviral regimen selected and recommended by these reference physicians for 100 salvage patients (second or subsequent failure) treated in the Brazilian public system (10 cases/doctor) for whom genotyping was being performed for the first time. The study was conducted in the following steps. It should be pointed out that at the time this study was conducted, nonpeptidic PIs, integrase inhibitors, and CCR5 antagonists were not a choice for salvage therapy, since this drugs were not available in Brazil.

\section{Step 1: Recording patient history}

Reference physicians recorded patient histories, including both clinical and treatment histories, in an electronic record format.

\section{Step 2: Baseline evaluation, treatment recommendation and reference physicians self-reported observations}

Reference physicians evaluated the patients' medical history reports, including antiretroviral exposures, histories of intol- erance, side effects, allergic reactions, viral loads and T cell counts. After this evaluation, the reference physicians were asked to empirically choose a preferred treatment option for the patients. Reference physicians were asked to summarize the bases on which they provided their recommended treatment options; these responses were recorded in the form of a self-completed electronic questionnaire.

Step 3: Genotype resistance test evaluation, treatment recommendation and reference physicians self-reported observations

Following the completion of Step 2, reference physicians received genotyping reports (ViroSeq 2.6). Upon receipt of these reports, the reference physicians again made treatment recommendations based on these first resistance test results and provided their recommended treatment options in the form of a self-completed electronic anamnesis.

Step 4: Virtual phenotype sequential resistance test evaluation, treatment recommendation and reference physicians self-reported observations

Following the completion of Step 3, reference physicians received virtual phenotype reports (VircoTYPE HIV-1 3.9.00) and again made treatment recommendations based on these resistance test results; the physicians also recorded the reasons for their final recommendations. The rationale for offering the virtual phenotype results after the genotyping results was the hypothesis that the more quantified assessments of resistance (i.e., the calculated fold change $v i s-\grave{a}$-vis the clinical cutoffs) provided by the virtual phenotypes would provide added value in this particular population of patients in which extensive resistance is expected to have occurred.

Finally, the reference physicians provided (via electronic self-completed anamnesis.) summaries of the added value offered by each report they had received during the study. This summary used a 4-point qualitative assessment scale to evaluate the utility of each resistance interpretation system in helping to select a new drug regimen.

\section{Statistical analyses}

To compare the number of active drugs selected for each regimen choice, the activities of the selected drugs were scored using a continuous phenotypic sensitivity score (cPSS) calculated as the sum of the activities of each drug in the regimen. Each drug was given a score of 1 if the phenotypic fold-change (FC) was less than the virtual phenotype lower clinical cutoff (CCO1), 0 if the FC was greater than the higher clinical cutoff ( $\mathrm{CCO} 2)$, or a value between 0 and 1 if the FC was between clinical cutoffs; where clinical cutoffs were not available for a particular drug, a cPSS score of 1 was used for FCs less than the biologic cutoff (BCO) and 0 for FCs greater than the BCO. 


\section{RESULTS}

\section{Demographics and treatment history}

The patients selected for this study were $53 \%$ male, and the median age was 37 years. The selected patients had undergone a median of three antiretroviral regimens over an average period of 5.2 years. The average number of drugs used during this period was 5.7. The average CD4 count was 356 cells $/ \mathrm{mm}^{3}$ (ranging from 4 to 2,072), and the average viral load was 198,157 copies/mL $\left(5.3 \log _{10}\right)$.

\section{Resistance profiles of patients' viruses}

The resistance profile of each patient's virus was determined from the latest genotype available for the patient (the same genotype used in Step 2 of the study). Patients had a median

Figure 1: Prevalence of mutations in the group of analyzed patients. Panel A depicts the Nucleoside Analog Reverse Transcriptase Inhibitors (NRTI) mutations, Panel B the Nonnucleoside Analog Reverse Transcriptase inhibitors (NNRTI) mutations, and panel $\mathrm{C}$ the protease inhibitors (PI) mutations. TAM: thymidine analog mutations.

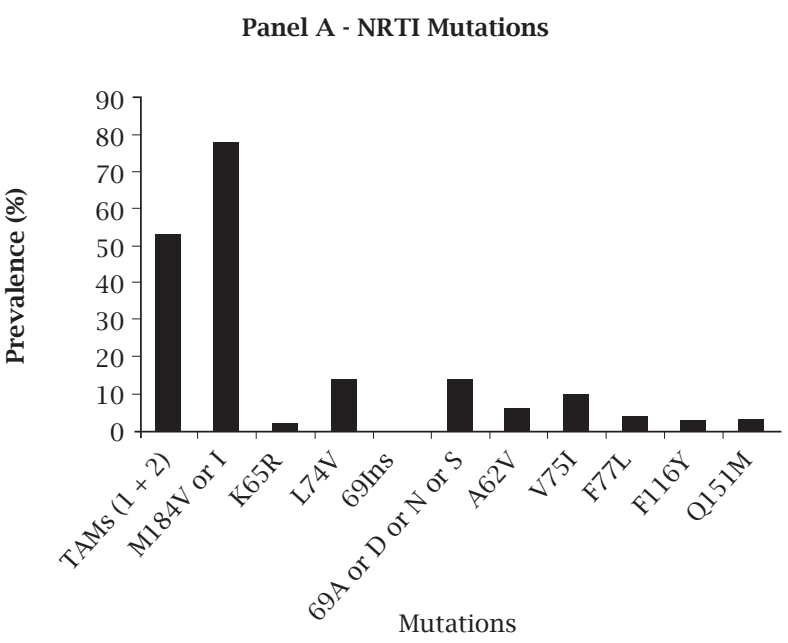

number of four NRTI mutations, with 52\% having any thymidine analog mutation (TAM), 78\% having $184 \mathrm{I} / \mathrm{V}$, and $12 \%$ having $74 \mathrm{~V}$ (Figure 1). A median of one NNRTI mutation was identified among the patients, with $42 \%$ having $103 \mathrm{~N}$ and $24 \%$ having 190G/S (Figure 1). Patients had a median number of two primary PI mutations, with $47 \%$ having $46 \mathrm{I} / \mathrm{L}, 42 \%$ having a substitution at codon 82 , and $40 \%$ having $90 \mathrm{M}$ (Figure 1).

\section{Changes in regimen selection following resistance interpretation}

In $79 \%$ of the 100 cases, the reference physicians changed their empirical choice of regimen after receiving the genotype (Table 1). Following subsequent receipt and consideration of a virtual phenotype report, reference physicians made additional regimen changes in $75 \%$ of the

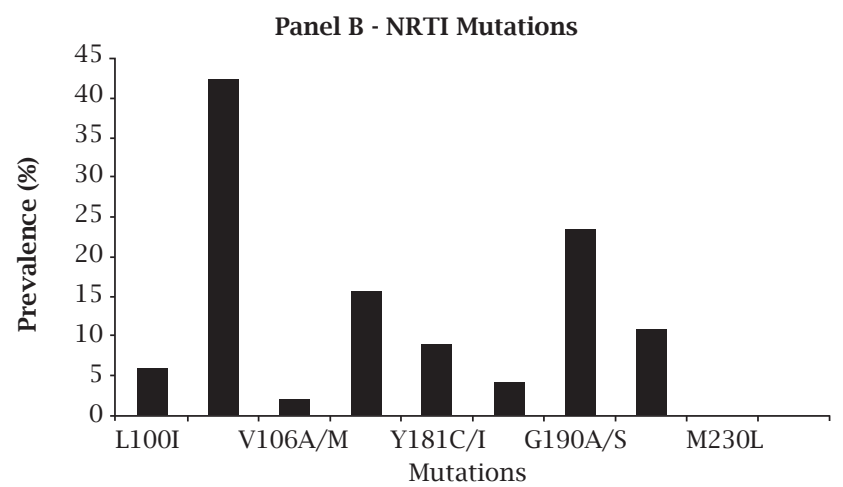

Panel C - NRTI Mutations

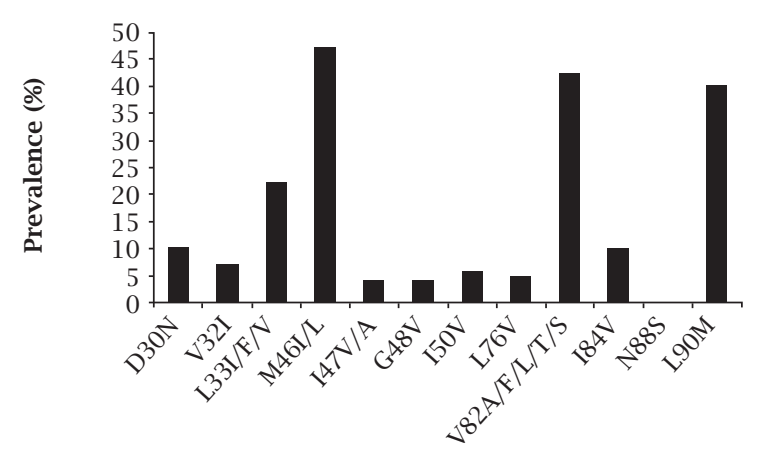

Mutations

Table 1. Percentage of change in the regimen, number of active drugs according to the phenotypic susceptibility score (PSS), and average number of drugs suggested by reference physicians, in each phase of the study

\begin{tabular}{lcccc}
\hline & $\begin{array}{c}\text { Changed } \\
\text { regimen } \\
\text { selection }\end{array}$ & $\begin{array}{c}\text { Number of } \\
\text { active drugs }\end{array}$ & $\begin{array}{c}\text { Total \# drugs } \\
\text { in regimen }\end{array}$ & p value \\
\hline $\begin{array}{l}\text { Phase I - no } \\
\text { resistance test }\end{array}$ & $79 \%$ & 1.8 & 4 & $\mathrm{p}=0.0004$ \\
\hline $\begin{array}{l}\text { Phase II - genotype } \\
\text { report }\end{array}$ & $75 \%$ & 2.2 & 4 & $\mathrm{p}>0.0001$ \\
\hline $\begin{array}{l}\text { Phase III - virtual } \\
\text { phenotype report }\end{array}$ & 2.8 & 4 & \\
\hline
\end{tabular}


patient cases. Categorization of regimen changes by ARV drug class showed that following receipt of the genotype, the highest proportion of switches were made to or from protease inhibitors $(60 \%)$ followed by changes to or from NRTIs (48\%), with the least number of switches being made to or from NNRTIs (29\%). Following subsequent receipt of the virtual phenotype, additional NRTI changes were made in $61 \%$ of regimens, additional PI changes in $49 \%$ of regimens, and NNRTIs were again changed the least (10\%). Therefore, the genotype report had a higher impact on the choice of PI and the virtual phenotype report had a higher impact on the choice of NRTI. Changes to a class were counted under any of the following conditions: replacement of 1 or more drugs by 1 or more different drugs of the same class, deletion of any drug from the class, or addition of 1 or more drugs from a previously non-selected class.

\section{Changes in individual drug selection}

The drugs most frequently used for salvage therapy were TDF (20\%), 3TC (17\%), LPV/r (16\%), EFV (9\%) and AZT $(6 \%)$. The drugs most frequently changed based on genotype were LPV/r and TDF, which together accounted for $16.7 \%$ of genotype changes, and the ratio of deletions to additions for both was approximately 2:1 (Table 2 ). The next

Table 2. Drugs most frequently changed

\begin{tabular}{|c|c|c|c|c|c|c|}
\hline \multirow{2}{*}{$\begin{array}{l}\text { All drug changes } \\
\text { including deletions } \\
\text { from and additions } \\
\text { to a regimen }\end{array}$} & \multicolumn{3}{|c|}{ Following genotype } & \multicolumn{3}{|c|}{ Following virtual phenotype } \\
\hline & $\mathrm{n}=216$ & $\mathrm{n}=216$ & $\mathrm{n}=216$ & $n=235$ & $n=235$ & $\mathbf{n}=235$ \\
\hline NRTIs & $\begin{array}{c}\text { All } \\
\text { changes }\end{array}$ & $\begin{array}{c}\text { Changes } \\
\text { from }\end{array}$ & $\begin{array}{c}\text { Changes } \\
\text { to }\end{array}$ & $\begin{array}{c}\text { All } \\
\text { changes }\end{array}$ & $\begin{array}{l}\text { Changes } \\
\text { from }\end{array}$ & $\begin{array}{c}\text { Changes } \\
\text { to }\end{array}$ \\
\hline AZT & $11.6 \%$ & $9.3 \%$ & $2.3 \%$ & $12.8 \%$ & $8.5 \%$ & $4.3 \%$ \\
\hline $3 \mathrm{TC}$ & $14.4 \%$ & $11.6 \%$ & $2.8 \%$ & $13.2 \%$ & $11.5 \%$ & $1.7 \%$ \\
\hline ddI & $6.9 \%$ & $4.6 \%$ & $2.3 \%$ & $18.3 \%$ & $9.4 \%$ & $8.9 \%$ \\
\hline $\mathrm{d} 4 \mathrm{~T}$ & $5.1 \%$ & $3.7 \%$ & $1.4 \%$ & $26.8 \%$ & $13.6 \%$ & $13.2 \%$ \\
\hline $\mathrm{ABC}$ & $8.8 \%$ & $5.6 \%$ & $3.2 \%$ & $7.7 \%$ & $4.7 \%$ & $3.0 \%$ \\
\hline FTC & $0.5 \%$ & $0.5 \%$ & $0.0 \%$ & $0.4 \%$ & $0.4 \%$ & $0.0 \%$ \\
\hline TDF & $16.7 \%$ & $11.6 \%$ & $5.1 \%$ & $14.0 \%$ & $11.1 \%$ & $3.0 \%$ \\
\hline \multicolumn{7}{|l|}{ NNRTIs } \\
\hline EFV & $9.7 \%$ & $5.6 \%$ & $4.2 \%$ & $3.4 \%$ & $1.3 \%$ & $2.1 \%$ \\
\hline NVP & $1.4 \%$ & $0.9 \%$ & $0.5 \%$ & $0.0 \%$ & $0.0 \%$ & $0.0 \%$ \\
\hline DLV & $2.3 \%$ & $0.0 \%$ & $2.3 \%$ & $1.3 \%$ & $0.9 \%$ & $0.4 \%$ \\
\hline \multicolumn{7}{|l|}{ PIs } \\
\hline IDV & $0.9 \%$ & $0.9 \%$ & $0.0 \%$ & $0.4 \%$ & $0.0 \%$ & $0.4 \%$ \\
\hline $\mathrm{IDV} / \mathrm{r}$ & $0.0 \%$ & $0.0 \%$ & $0.0 \%$ & $0.4 \%$ & $0.0 \%$ & $0.4 \%$ \\
\hline NFV & $1.9 \%$ & $1.4 \%$ & $0.5 \%$ & $0.0 \%$ & $0.0 \%$ & $0.0 \%$ \\
\hline SQV & $6.5 \%$ & $2.3 \%$ & $4.2 \%$ & $6.4 \%$ & $4.7 \%$ & $1.7 \%$ \\
\hline $\mathrm{SQV} / \mathrm{r}$ & $0.0 \%$ & $0.0 \%$ & $0.0 \%$ & $2.6 \%$ & $0.4 \%$ & $2.1 \%$ \\
\hline APV & $0.0 \%$ & $0.0 \%$ & $0.0 \%$ & $0.0 \%$ & $0.0 \%$ & $0.0 \%$ \\
\hline $\mathrm{APV} / \mathrm{r}$ & $2.3 \%$ & $0.0 \%$ & $2.3 \%$ & $4.3 \%$ & $1.7 \%$ & $2.6 \%$ \\
\hline fAPV & $0.5 \%$ & $0.5 \%$ & $0.0 \%$ & $0.0 \%$ & $0.0 \%$ & $0.0 \%$ \\
\hline $\mathrm{fAPV} / \mathrm{r}$ & $1.9 \%$ & $1.4 \%$ & $0.5 \%$ & $0.9 \%$ & $0.4 \%$ & $0.4 \%$ \\
\hline $\mathrm{LPV} / \mathrm{r}$ & $16.7 \%$ & $10.2 \%$ & $6.5 \%$ & $10.6 \%$ & $5.5 \%$ & $5.1 \%$ \\
\hline ATV & $0.5 \%$ & $0.0 \%$ & $0.5 \%$ & $0.0 \%$ & $0.0 \%$ & $0.0 \%$ \\
\hline $\mathrm{ATV} / \mathrm{r}$ & $2.3 \%$ & $1.4 \%$ & $0.9 \%$ & $2.6 \%$ & $0.9 \%$ & $1.7 \%$ \\
\hline \multirow{2}{*}{$\mathrm{TPV} / \mathrm{r}$} & $6.5 \%$ & $0.0 \%$ & $6.5 \%$ & $8.1 \%$ & $4.7 \%$ & $3.4 \%$ \\
\hline & $100 \%$ & $100 \%$ & $100 \%$ & $100 \%$ & $100 \%$ & $100 \%$ \\
\hline
\end{tabular}


most frequently changed drugs based on the genotype report were 3TC (14.4\%) and AZT (11.6\%), and again deletions from the regimen outnumbered additions. Following the virtual phenotype report, $\mathrm{d} 4 \mathrm{~T}$ (26.8\%) and ddI (18.3\%) were the drugs most frequently changed, and in both cases, deletions from the regimen marginally outnumbered additions. These drugs were followed by $3 \mathrm{TC}$ (13.2\%) and AZT (12.8\%). Darunavir was not an option for the reference physicians, since it was not registered at the time the study was conducted. Enfuvirtide was a choice in $17 \%$ of the empirically based regimens, in $38 \%$ of the genotype-based regimens, and in $34 \%$ of the virtual phenotype-based regimens. In seven cases where Enfuvirtide was chosen on the basis of the genotype report, the physician decided to choose an antiretroviral other than Enfuvirtide after receiving the virtual phenotype report.

\section{Results of changes in regimen selection}

The regimen changes that occurred after receipt of the genotype report resulted in an increase in the mean number of active drugs from 1.8 to $2.2(\mathrm{p}=0.0003)$, while the total number of drugs selected remained at a mean of 4.0. Subsequent changes following consideration of the virtual phenotype report resulted in a further increase in the number of active drugs from 2.2 to 2.8 $(\mathrm{p}<0.0001)$, while the mean number of drugs per regimen remained at 4.0 .

\section{Treatment cost after regimen selection}

Some of the available drugs in Brazil are generic and represent a lower cost for the government. Non-generic drugs include Abacavir, Tenofovir, Lopinavir, Atazanavir, Amprenavir, Nelfinavir, and Enfuvirtide. The average costs of empirically based, genotype-based and virtual phenotype-based salvage therapies were US $\$ 20.16$, US $\$ 31.99$ and US\$28.81/day, respectively (ANOVA: empirical $v s$. genotype, $\mathrm{p}<0.001$; empirical $v s$. virtual phenotype, $\mathrm{p}<0.001$; genotype $v s$. virtual phenotype, non-significant). The individual costs of the drugs in Brazil can be seen at www.aids.gov.br.

\section{Physician assessment of the utility of the resist- ance interpretations}

Following consideration of the genotypic interpretations and subsequently of the virtual phenotypes, the reference physicians assessed the utility of each in helping to select treatment options (Table 3). The majority of the expert physicians found the genotypic information to be useful ( $88 \%$, extremely/very/moderately useful). The majority $(84 \%)$ also found the subsequent virtual phenotype to be useful. Fourteen percent of the reference physicians rated the genotype as "extremely useful", whereas $34 \%$ rated the subsequent virtual phenotype as "extremely useful" ( $p=0.0003)$.
Table 3. Utility of the resistance tests according to the reference physicians

\begin{tabular}{lcc}
\hline & Genotype & $\begin{array}{c}\text { Virtual } \\
\text { phenotype }\end{array}$ \\
\hline Extremely useful & $13 \%$ & $34 \%$ \\
\hline Very useful & $51 \%$ & $25 \%$ \\
\hline Moderately useful & $23 \%$ & $25 \%$ \\
\hline Did not make any difference & $13 \%$ & $16 \%$ \\
\hline Total & $100 \%$ & $100 \%$ \\
\hline
\end{tabular}

\section{DISCUSSION}

In this study we analyzed the impact of resistance test interpretations on medical opinion in HIV-1 salvage therapy. For this purpose, we used ten very experienced Brazilian reference physicians, all well-trained to provide HIV salvage therapy guidance and genotypic resistance interpretation to attending physicians within the Brazilian public system. We found that resistance test interpretations played a key role in influencing the opinions of this group of physicians: $79 \%$ of the time they chose a different antiretroviral after analyzing the genotypic profile of the patient's virus and $75 \%$ of the time the group further changed their drug selection when a virtual phenotype interpretation was offered after the genotype report. We believe that the phenotype-based report may further influence the physician's decision (especially in cases where extensive resistance is documented in the genotype result) due to the quantitative nature of the phenotype result and the presence of clinical cutoffs. It is possible that the quantitative aspect of the phenotypic interpretation (given by the fold change values) enables physicians to build a more active antiretroviral backbone, as drugs with values closer to the lower cutoff tend to be chosen. Indeed, genotypic resistance testing, phenotypic resistance testing and virtual phenotypes ${ }^{9,12,13}$ have been proven to be effective for optimizing salvage therapy regimens and to provide a better virologic response than standard care. It is interesting to note that virtual phenotypes have been found to provide similar or even better salvage regimens when compared to conventional phenotype tests. ${ }^{12,13}$

It has been shown that the PSS correlates well with virologic response to salvage therapy, with the number of active drugs being proportional to the decline in viral load..$^{14}$ For this reason, we used the PSS to calculate the average number of active drugs prescribed in each study phase. Interestingly, the use of a resistance test increased the number of active drugs, and use of the virtual phenotype resulted in a still higher average number of active drugs (2.8). It is of note that the average number of drugs available for use in a regimen was the same for each study phase, and thus, the increase in 
the number of active drugs was not due to an increase in the number of available antiretrovirals, but rather to a tendency to select drugs with more activity. In spite of the higher cost of the antiretrovirals prescribed after resistance tests in this study, the increase in potential virologic efficacy with the use of more active and durable regimens may conceivably make salvage therapy guided by resistance testing cost-effective, as has been previously described..$^{15}$

One caveat of this study is that the reference physicians analyzed patients followed by other attending physicians instead of patients followed by them. Therefore, although the reference physicians used all of the available clinical data and data about antiretroviral tolerance, side effects and allergies for each case, it is possible that in the real world, some of the suggested drugs or combinations of drugs would not be used. Thus, we acknowledge that although there is a significant impact of resistance test interpretation on physicians' decision-making, this effect may not translate into a large change in the choice of drugs. Therefore, the increase in the number of active drugs prescribed may be overestimated in this study compared to what would happen in real life.

Nonetheless, it has been shown that resistance tests not only positively impact virologic outcome but also impact patients' survival, ${ }^{16}$ and these impacts clearly result from the influence of resistance test interpretations on the choice of antiretrovirals prescribed by physicians providing care to HIV-infected patients.

\section{ACKNOWLEDGEMENTS}

We are indebted to PN-DST/AIDS of the Brazilian Ministry of Health.

\section{REFERENCES}

1. Deeks SG, Hoh R, Neilands TB et al. Interruption of treatment with individual therapeutic drug classes in adults with multidrug-resistant HIV-1 infection. J Infect Dis 2005; 192:1537-44.

2. Medeiros R, Diaz RS, Filho AC. Estimating the length of the first antiretroviral therapy regimen durability in São Paulo, Brazil. Braz J Infect Dis 2002; 6:298-304.

3. Napravnik S, Edwards D, Stewart P, Stalzer B, Matteson E, Eron Jr JJ. HIV-1 drug resistance evolution among patients on potent combination antiretroviral therapy with detectable viremia. J Acquir Immune Defic Syndr 2005; 40:34-40.
4. Sucupira MC, Souza IE, Costa LJ, Scheinberg MA, Diaz RS. Antiretroviral treatment failure and HIV-1 genotypic resistance in São Paulo, Brazil. Antivir Ther 2001; 6:263-4.

5. Lohse N, Kronborg G, Gerstoft J et al. Virological control during the first 6-18 months after initiating highly active antiretroviral therapy as a predictor for outcome in HIV-infected patients: a Danish, population-based, 6-year follow-up study. Clin Infect Dis 2006; 42:136-44

6. Zaccarelli M, Tozzi V, Lorenzini $\mathrm{P}$ et al. Multiple drug classwide resistance associated with poorer survival after treatment failure in a cohort of HIV-infected patients. Aids 2005; 19:1081-9.

7. Durant J, Clevenbergh P, Halfon $\mathrm{P}$ et al. Drug-resistance genotyping in HIV-1 therapy: the VIRADAPT randomised controlled trial. Lancet 1999; 353:2195-9.

8. Baxter JD, Mayers DL, Wentworth DN et al. A randomized study of antiretroviral management based on plasma genotypic antiretroviral resistance testing in patients failing therapy. CPCRA 046 Study Team for the Terry Beirn Community Programs for Clinical Research on AIDS. Aids 2000; 14:F83-93.

9. Cohen CJ, Hunt S, Sension $M$ et al. A randomized trial assessing the impact of phenotypic resistance testing on antiretroviral therapy. Aids 2002; 16:579-88.

10. Tural C, Ruiz L, Holtzer C et al. Clinical utility of HIV-1 genotyping and expert advice: the Havana trial. Aids 2002; 16:209-18.

11. Vray M, Meynard JL, Dalban C et al. Predictors of the virological response to a change in the antiretroviral treatment regimen in HIV-1-infected patients enrolled in a randomized trial comparing genotyping, phenotyping and standard of care (Narval trial, ANRS 088). Antivir Ther 2003; 8:427-34.

12. Mazzotta F, Lo Caputo S, Torti $C$ et al. Real versus virtual phenotype to guide treatment in heavily pretreated patients: 48-week follow-up of the Genotipo-Fenotipo di Resistenza (GenPheRex) trial. J Acquir Immune Defic Syndr 2003; 32:268-80.

13. Perez-Elias MJ, Garcia-Arota I, Munoz V et al. Phenotype or virtual phenotype for choosing antiretroviral therapy after failure: a prospective, randomized study. Antivir Ther 2003; 8:577-84.

14. Swanstrom R, Bosch RJ, Katzenstein D et al. Weighted phenotypic susceptibility scores are predictive of the HIV-1 RNA response in protease inhibitor-experienced HIV-1-infected subjects. J Infect Dis 2004; 190:886-93.

15. Weinstein MC, Goldie SJ, Losina E et al. Use of genotypic resistance testing to guide HIV therapy: clinical impact and cost-effectiveness. Ann Intern Med 2001; 134:440-50.

16. Palella F, Armon C, Chmiel J et al. Enhanced Survival Associated with Use of HIV Susceptibility Testing among HAARTexperienced Patients in the HIV Outpatient Study (HOPS) $13^{\text {th }}$ Conference on Retroviruses and Opportunistic Infections. Denver, CO, 5-8 February 2006. 